This is an electronic reprint of the original article. This reprint may differ from the original in pagination and typographic detail.

Author(s): Collin, Kaija; Herranen, Sanna; Valleala, Ulla Maija; Paloniemi, Susanna

Title: Interprofessional collaboration during an emergency ward's rounds

Year: $\quad 2015$

Version:

Please cite the original version:

Collin, K., Herranen, S., Valleala, U. M., \& Paloniemi, S. (2015). Interprofessional collaboration during an emergency ward's rounds. International Journal of Emergency Services, 4(2), 212-226. https://doi.org/10.1108/IJES-02-2015-0007

All material supplied via JYX is protected by copyright and other intellectual property rights, and duplication or sale of all or part of any of the repository collections is not permitted, except that material may be duplicated by you for your research use or educational purposes in electronic or print form. You must obtain permission for any other use. Electronic or print copies may not be offered, whether for sale or otherwise to anyone who is not an authorised user. 


\section{Interprofessional Collaboration during an Emergency Ward's Rounds}

Purpose - This case study explores interprofessional collaboration during ward rounds on a Finnish emergency and infection ward from the viewpoint of three central professional groups: physicians, nurses and secretaries.

Design/methodology/approach - We utilise an ethnographically informed approach, with observations and interviews as the data collection devices. The data comprise ten interviews with staff members and ten hours of observations. The data were analysed using qualitative thematic analysis.

Findings - The ward rounds were found to be rather physician- and medicine-centred, and mostly not interprofessional. Nurses and secretaries in particular expressed dissatisfaction with many of the current ward rounds work practices. Ward rounds are an essential part of collaboration in implementing the emergency-natured operational aim of the ward, yet we found that the ward rounds are complicated by diverging professional views and expectations, variable work practices and interactional inequality.

Originality/value - This study makes a contribution to the research of collaboration in emergency care and ward rounds, both of which are little-studied fields. Further, contextspecific studies of collaboration have been called for in order to eventually create a model of shared expertise. The findings of this study can be utilised in studying and developing emergency care contexts.

\section{Introduction}

Interprofessional collaboration

Tasks in health care are becoming more and more intricate. A demand for even better collaboration among professionals has thus arisen (Hoskins, 2012), as it has been seen to improve the quality of health care (MacNaughton et al., 2013), in particular, patient safety (Baker et al., 2006) and patient-based, holistic care (McCallin, 2001; D’Amour et al., 2005; Baker et al., 2006). Characteristic of interprofessional collaboration is that work, its practices and the object of work are examined as a whole, combining expertise from different professional fields in a work community and work group (Housley, 2003). Interprofessionalism has thus also been called shared and collegial expertise. Patient care is a task shared among professionals, which posits that tasks and collaboration are synchronised (Baker et al., 2006). However, such ideals are rarely present in the reality of health care. Instead, the realisation of interprofessional collaboration in different health care contexts varies; the degree of interprofessional collaboration depends on organisations and work tasks (Millward and Jeffries, 2001; Collin et al., 2012). 
In this case study, our aim is to explore interprofessional collaboration in an emergency care context, more specifically during ward rounds, from the viewpoints of three central professional groups: physicians, nurses and secretaries. Various local and context-specific studies of interprofessional collaboration have been called for in order to eventually create a general model of shared expertise (Collin et al., 2015), to which this study aims to make a contribution. Further, the operational aim of the ward studied here is that patients' stay there should not exceed two days; within this time frame, patients should either be transferred to longer-stay wards or discharged. Ward rounds are an essential means of collaboration in realising this operational aim.

Interprofessionalism is not always present in health care work, nor is it necessarily required at all times. A study concerning an emergency clinic (Collin et al., 2012) found that the majority of the work can be most suitably carried out through multi-professional collaboration: professionals working together and exchanging information but still strongly keeping to their own professional groups. Interprofessionalism was found to arise particularly in the most challenging parts of work processes, such as when dealing with patients with lifethreatening conditions. It was concluded (Collin et al., 2015) that in such highly challenging work situations, professional hierarchies are temporarily dropped and individual professional experience and know-how are more valuable.

Major obstacles to the implementation of collaboration in health care have been found to stem from hierarchic organisation and administration (Ramanujam and Rousseau, 2006). Furthermore, health care professionals are indeed educated and managed professionspecifically, yet expected to collaborate seamlessly in practice (Wilbur, 2014). Rigid and hierarchic health care decision-making prevents collegial decision-making, particularly in stressful situations, and impedes the flow of information and interaction between different professional groups and levels of decision-making (Nembhard and Edmondson, 2006; see also Pisano et al., 2001). Indeed, interprofessional collaboration is often realised at a junction of diverging interests and expectations (Copnell et al., 2004; Krogstad et al., 2004), owing to the differing tasks of professional groups regarding patients and to official or perceived professional status (Krogstad et al., 2004). Problems of hierarchy can be alleviated by developing teamwork and interaction between professional groups (Baker et al., 2006). In particular, developing mutual trust and an open work climate have been found to be important for well-functioning interprofessional collaboration (San Martín-Rodriguez et al., 2005; Baker et al., 2006).

\section{Ward rounds}

Ward rounds are a pivotal part of care planning, education and collaboration of different professional groups in hospitals (Creamer et al., 2010; Fiddler et al., 2010), but despite their long tradition, they have not been much studied (Creamer et al., 2010). The convention of executing ward rounds has varied from a meeting between staff members in a conference 
room to traditional bedside rounds (Walton and Steinert, 2010). Although ward rounds are, essentially, opportunities for interprofessionalism, in practice their implementation varies and they are often dominated by (senior) physicians (Weber et al., 2007; Walton and Steinert, 2010). In emergency care, time is of the essence, and smooth collaboration during ward rounds is thus also indispensable.

On the one hand, the traditional, physician-dominated ward round has been seen to bring safety and structure to the work; on the other hand, it is rigid and prevents efficient collaboration (Fiddler et al., 2010). Nurses, for example, have expressed discontentment with ward rounds due to their often minimal role in them (e.g. Manias and Street, 2001). Efficient collaboration is evidently linked to better patient outcomes, yet the often physician-centred culture of ward rounds has been found to exclude other professionals and prevent them from providing their clinical input. Senior physicians are in a key position in hospital change processes with regard to improving teamwork (Bradfield, 2010), including in the context of ward rounds.

Ward rounds have typically been viewed as occasions for informing patients, but at their most ideal they could function as opportunities for fruitful interaction between patients and staff members as well as between different professional groups. Ward rounds are often the sole occasions where physicians, nurses and patients can discuss care aims or discharge, for example (Weber et al., 2007). In a few studies, interventions have been implemented to develop ward rounds in a more interprofessional direction, and experiences of these interventions have been examined with surveys (O’Leary et al., 2010) and interviews (Fiddler et al., 2010). Interprofessional ward rounds have, in fact, yielded positive results: for instance, the deployment of a structured form focussed on the central issues in ward rounds, as a tool to enhance interprofessional communication, was found to improve collaboration significantly (O’Leary et al., 2010).

Studies concerning ward rounds have been conducted on different kinds of wards, including critical care (e.g. Manias and Street, 2001), where communication was found to be quite physician-dominated. When nurses had the opportunity to speak, they gave information about patients and asked questions on behalf of the patients or their families. It can be seen that ward rounds form a central interprofessional work practice involving not only professionals but also patients and their kin. In the next chapter, we introduce our research context and research aim regarding interprofessional collaboration and ward rounds.

\section{Research context, aim and research questions}

Research on both collaboration in emergency care (Lemieux-Charles and McGuire, 2006) and ward rounds (Creamer et al., 2010) is still rather meagre. This case study aims to contribute to filling these gaps by exploring ward rounds on an emergency and infection ward (EIW) with 41 beds and approximately 56 staff members. The study is part of a larger research and development project on teamwork and interprofessional collaboration (INPROF) conducted between 2010 and 2012 in a major emergency unit in Finland. The unit 
comprises an outpatient clinic and the EIW, which work in close collaboration - the EIW receives virtually all of its patients from the clinic - and the total number of emergency unit staff is between 170 and 200. The aim of INPROF was to study emergency work processes, well-functioning work practices and problems in terms of interprofessional collaboration, in close cooperation with the staff of the unit.

During the study, the EIW saw patients belonging to all medical specialities and was therefore subject to multiple and often overlapping ward rounds (surgery, internal medicine, neurology, gynaecology and lung diseases). The three last-mentioned specialities often had smaller-scale ward rounds in the sense that the EIW did not always have patients belonging primarily to these specialities: in these cases, medical specialists from other units of the hospital would come to the EIW for ward rounds. Specific professionals frequently participated in the ward rounds on the EIW, so there was not a great turnover in terms of ward round staff. Various physicians and other professionals (e.g. social workers) from other wards did visit the EIW for consultations, but in this study we wanted to focus on the collaboration of the permanent EIW professionals, as this had emerged as a problematic area. All of the ward rounds on the EIW were traditional bedside rounds. The operational aim of the EIW is that patients' stay there should not exceed two days; within this time frame, patients should either be transferred to longer-stay wards or discharged. Ward rounds are an essential part of realising this operational aim.

The aim of this study is to examine interprofessional collaboration in ward rounds from the viewpoint of three central professional groups: physicians, nurses and secretaries. These were the three professional groups whose members participated in ward rounds on the EIW, which is why they were scrutinised in this study. The research questions are as follows:

1) What are the main contents and challenges of ward rounds from the viewpoint of the different professional groups' work?

2) What kinds of expectations do the different professionals have of interprofessional collaboration on ward rounds?

3) What kinds of differences are there in the professionals' actions and perceptions concerning interprofessional collaboration on ward rounds?

Neither this study nor the larger project was targeted at patients. A research permit for the project was acquired from the ethical committees of both the university and the hospital. In addition, consent was obtained from individuals who participated. The hospital and ward participating in the study were disclosed in research publications based on a mutual agreement. Identifying information regarding the participants in the following data extracts has been removed to protect anonymity.

\section{Methods}

\section{Data collection}


The methodological approach in this study was informed by ethnography and collective ethnography (e.g. Brewer, 2000; Woods et al., 2000; Gordon et al., 2006; Sigaud, 2008), which enable a profound understanding of the research target and context as well as quick changes in data collection procedures if necessary, which are well-suited to an emergency work context. Altogether four researchers participated in the project. The data collection methods were observations in situ, in natural work situations; and individual and focus group interviews.

'Informed by ethnography' denotes here that the data collection was ethnographic, as it involved observations. We acknowledge that a complete ethnographic methodology would have demanded a longer period of observations of the ward rounds and more extensive data (e.g. more professionals being observed during the ward rounds). We did, however, have a good understanding of the entire research context (the emergency unit), as in the INPROF project, as the first step, we aimed to acquaint ourselves with the context by observing everyday work in the emergency unit. The data from these observations comprised a total of 85 hours of observations and 82 pages of field notes; this amount of data appeared sufficient, as similar findings were already repeated (Patton, 2002) and, based on the data, especially problematic areas of collaboration (e.g. the ward rounds) could be fluently determined for closer study together with the staff of the unit. Even though these data were mainly from the outpatient clinic and were not analysed for this particular study, they gave us a good understanding of the context, since the clinic and the EIW work in close collaboration. This understanding generally facilitated the study of different sub-themes, such as ward rounds, in the project. Aside from the contextual knowledge, discussions in the steering group of the project (the managers of the unit and the researchers) directed us to focus specifically on ward rounds in terms of the EIW. This grounding - an understanding of the nature and challenges of collaboration in this context - also served as a helpful interpretational framework.

Ward rounds were observed for approximately ten hours, two researchers at a time, and documentation methods during observations included field notes and audio recordings. As for the field notes, the purpose was to characterise the contents of ward rounds, instances of collaboration and interactions between professionals in order to understand ward round work practices and analyse interprofessional collaboration. Audio recording was used to document verbal communication between professionals in situations of collaboration.

The interview themes comprised the contents of ward rounds, perceptions of the current state of ward rounds, challenges, developmental ideas and expectations of collaboration. Through these themes, we wanted to examine whether the different professionals shared an understanding of collaboration, which is a fundamental precondition for interprofessionalism (D’Amour et al., 2005; San Martín-Rodriguez et al., 2005). Ten members of the staff participated in the interviews: nurses $(n=5)$, secretaries $(n=3)$ and physicians $(n=2)$. The interviews were audio recorded (altogether about 210 minutes, 78 pages of transcriptions). The interviewees were professionals frequently participating in the ward rounds on the EIW, thus, they were key informants (Patton, 2002) from all the relevant professional groups. The 
two physicians and one nurse were interviewed individually, and the other participants were interviewed as two focus groups (two nurses and three secretaries). The reason for focus group interviews was practical, relating to the three-shift work on the EIW.

The interviewees and observees were mostly the same individuals, but naturally other staff members were also observed during ward rounds (the number of observees is altogether about 30). All of the interviewed physicians and nurses were observed, but not all of the interviewed secretaries could be observed due to schedule conflicts. Further, ward rounds of all the different medical specialities were observed. Professionals who participated in the ward rounds were medical specialists, nurses and, during some ward rounds, registrars. Secretaries participated in the ward rounds of only one medical speciality. The field data comprised about 17 pages of field diary notes, 120 minutes of audio recordings and 44 pages of transcriptions of these recordings. In addition, an audio recording from a staff development session (about 58 minutes, 201/2 pages of transcriptions) was utilised to complement the interviews and verify the observations made by the researchers. In terms of the data of this study, similar findings were already repeated (Patton, 2002), hence the amount of data seemed justified.

\section{Analysis}

The data from this study were analysed using qualitative thematic analysis (Braun and Clarke, 2006). To commence the analysis, the data were transferred to the ATLAS.ti software, which enabled the thematising of the data. First, the interviews were thematised: the purpose was to find contents, challenges, developmental ideas and expectations pertaining to ward rounds from the viewpoints of the different professional groups. After analysing the interviews, the observation data were analysed in a data-driven manner by thematising. In the final phase of the analysis, the perceptions and actions of the different professionals were compared to each other. The findings and interpretations for the first research question (contents and challenges of ward rounds) were based on both the observation and the interview data, and for the second research question (expectations of interprofessional collaboration), they were based more on the interview data. As for the third research question (differences in actions and views regarding interprofessional collaboration), the findings and interpretations were built on the findings from the first and second research questions.

\section{Findings}

In the following chapters, we present our findings both generally and pertaining to particular professional groups. The findings have been organised according to the three research questions, and we have also tried to indicate which findings are based on which data.

\section{The contents and challenges of ward rounds}

In the interviews, the physicians identified exchange of information and advancement of treatment processes as the most important contents of ward rounds. Further, a swift 
implementation of ward rounds was mentioned as a central content in order to formulate a care plan for patients and have them undergo the necessary tests as quickly as possible. Based on the interviews and observations, some physicians valued interprofessional collaboration, whereas other physicians did not regard the presence of nurses during ward rounds as important if the necessary information was available through other channels. In Extract 1, one of the interviewed physicians describes the main contents of ward rounds:

\section{Extract 1. A physician describing the main contents of ward rounds}

Physician: the point is to be able to transfer patients forward: to a long-stay ward or home. ... [During a ward round] there's a physician, a nurse and a patient present, possibly also next of kin, and together we go through the patient's situation.

Based on the observations, physicians did pursue interactions with patients during ward rounds and, in terms of the physicians' work, a ward round could sometimes be successfully carried out without nurses. However, implementing ward rounds without nurses was possible only when patients were in good enough health to communicate for themselves. According to the interviews and observations, the challenges of ward rounds from the physicians' viewpoint related largely to medicine and data systems. For example, a lack of up-to-date test results due to the early timing of ward rounds was mentioned as a problem. The ward medication data system was also seen as rigid and laborious. Based on the observations, during ward rounds physicians also attended to a variety of other tasks, such as phone consultations. This partly hampered the participation of nurses in ward rounds: physicians' other tasks were sometimes quite time-consuming, and nurses were not always able to wait

for a ward round to continue and had to leave for other duties. Extract 2 from the field notes depicts this:

\section{Extract 2. A physician attending to other tasks during a ward round}

During the ward round, this physician attends to a number of other tasks, too, e.g. consultations, which seems to make it more difficult for nurses to participate. Nurses are seemingly unable to be present throughout the whole ward round, because it would simply take too much of their time.

The interviewed nurses also identified exchange of information and advancement of treatment processes as the most central contents of ward rounds. Based on the interviews and observations, being able to participate in ward rounds was important from nurses' viewpoint for three reasons in particular: i) nurses have up-to-date and long-term information on patients' health and on the effectiveness of administered medication, and during ward rounds nurses receive updated care information from physicians, ii) during ward rounds, nurses serve as the 'voice' of patients towards physicians if patients are in poor health or are afraid to ask physicians questions directly, and iii) nurses can act as interpreters between physicians and patients, denoting that particularly after ward rounds, nurses are able to explain the impending treatment to patients more thoroughly if patients feel they need more information. Based on the observations, nurses implemented tasks pertaining to all of the above-mentioned 
contents during ward rounds. Extract 3 from the field notes depicts information exchange between a physician and a nurse:

\section{Extract 3. Information exchange between a physician and a nurse}

With this patient, the physician communicates directly; a nurse is not needed to "interpret" (apparently the discussion concerns cancer). They also talk about resuscitation. The nurse returns to follow the discussion between the physician and the patient. The physician asks the nurse how the resuscitation marking is transferred to the data systems, and receives instructions for this from the nurse. The nurse then asks the physician as to where the patient will be transferred.

The role of nurses was highlighted especially when patients were in poor health: in these cases, nurses participated in care planning more equally. Apart from care planning, some ward rounds seemed to include additional purposes: medical procedures were conducted and samples were taken. The presence of nurses was also important in these cases. In Extract 4, a nurse describes the main contents of ward rounds:

\section{Extract 4. A nurse describing the main contents of ward rounds}

Nurse: you receive updated information - what will happen to patients next. And you can interpret information to patients, since physicians' points sometimes remain unclear to them. ... During ward rounds you can inform physicians on patients' health. Sometimes patients are afraid to speak during ward rounds, so you're also kind of the 'voice' of patients in these cases.

Even though from the physicians' viewpoint some ward rounds could be successfully implemented without nurses, from the nurses' viewpoint, this kind of implementation complicates their work. The nurses indicated that ward rounds were an important information channel for them even when they did not actively participate in the discussion during the ward rounds. Nurses said that they sometimes observed and picked up crucial information from the conversation of the physicians. If nurses were unable to participate in ward rounds, finding this crucial information afterwards could prove difficult. A challenge identified by both the nurses and the secretaries was also that physicians did not usually phone nurses to inform them that a ward round was in progress; rather, nurses sought out the correct ward round on their own or asked the secretaries. A major issue identified by the nurses and the secretaries was also the overlapping of ward rounds and other duties. The role of these two professional groups regarding ward rounds on the EIW did, in fact, seem to involve a great deal of waiting and being on standby. On occasion, nurses solved the problem of overlaps by participating in ward rounds and attending to other duties in the same room, but naturally this was not always feasible.

According to the secretaries, the most essential content of ward rounds was the preparation of ward rounds, which denoted, for example, preparing the ward round carts (a computer and paper sheets containing patient and staff data). Secretaries also participated in the ward rounds of one medical speciality, in which case their task was mainly to phone nurses to alert them to join the ward round. Extract 5 from the field notes illustrates a secretary being on 
"standby" during a ward round and also expounds why the role of secretaries in the ward rounds often seemed futile to the secretaries themselves:

\section{Extract 5. A secretary and physicians during a ward round}

The secretary has not said anything during the entire ward round, except for now when one of the physicians asks about the next patient. Until now, the physicians participating in the ward round have checked the patients' information on the computer in a self-directed manner.

Apart from the previously mentioned challenges of overlaps and information breakdowns, the secretaries also identified long or late-arriving dictations of physicians' notes as a challenge because this often caused haste and 'traffic jams'. In Extract 6, two secretaries describe the contents and challenges of ward rounds:

\section{Extract 6. Two secretaries describing the contents and challenges of ward rounds}

Secretary 1: It's our job to make sure that nurses find the correct ward round, and that the ward round data sheets are in order.... Secretary 2: Sometimes the long dictations really trouble us.

The findings described above are summarised in Table 1.

[Table 1 here]

All of the professional groups basically participated and performed tasks related to their own professional group's duties during ward rounds but based on the observations and interviews there were differences in the nature of the interactions. These differences related, for example, to the consideration of fellow professionals' viewpoints and distinct features of different medical specialities: some medical specialities seemed to emphasise brevity in ward rounds, so conversations were rather short and concise overall, whereas some ward rounds were longer and more thorough in nature. We observed ward rounds of all the different medical specialities on the EIW, and Extract 7 from the field notes depicts a rather typical interaction during ward rounds:

\section{Extract 7. Interaction during a ward round}

A secretary states the next patient's room number (the physicians have a list of patients, but they're not looking at it; the secretary is running the situation). Physician 1 talks to the patient about the treatment. More examining takes place, in which a nurse assists. The nurse then asks whether food may be given to the patient and remains with the patient when the others leave the room. The next patient's nurse arrives and tells about the patient's health. The physicians are reading the patient's information on the computer and examining one of the imaging results. The ward round continues. In the next room, a nurse is apparently continuously present; also, this nurse is brisk and speaks concisely about the patient's health. In my opinion, however, this last nurse was the only one who tried to participate in the decision-making. However, physician 1 did not take the nurse's points into account in his decisions. 
Expectations regarding interprofessional collaboration in ward rounds

In the interviews, the physicians presumed that the other professional groups (nurses and secretaries) expected them to communicate with patients more thoroughly, and to give treatment orders during the ward rounds, not afterwards. In turn, the physicians expected upto-date information on patients' health and practicalities, as well as fluent collaboration. The nurses presumed that the other professional groups (physicians and secretaries) expected flexibility, fluent collaboration and precise implementation of treatment orders. Likewise, the nurses expected clear treatment orders and flexibility, such as understanding and reconciling overlaps in schedules. The secretaries presumed that the other professional groups (physicians and nurses) expected them to run the practicalities smoothly, which was greatly affected by the smooth exchange of information that the secretaries expected of the other professional groups. These findings are summarised in Table 2.

[Table 2 here]

Comparing the actions and views of the different professionals

Both the interviewed physicians and the interviewed nurses identified exchange of information and advancement of treatment processes as the most essential contents of ward rounds, yet in practice, and partly also in the interviews, different professionals seemed to have rather diverging views on what kinds of information and means of information were necessary. Physicians primarily wanted up-to-date and pertinent medical information through any channel. The presence of nurses during ward rounds was not seen as vital if the necessary information was otherwise available (electronically or from patients, for example). In Extract 8, a physician discusses this:

\section{Extract 8. A physician discusses nurses' role in terms of ward rounds}

Physician: It's not a big problem for physicians that nurses have to run there for ward rounds, it's probably nurses’ problem.

Nurses, on the other hand, wished (and, in practice, often strove) for more interactionally equal collaboration during ward rounds where both medical and non-medical information is exchanged. In Extract 9, a nurse discusses collaboration with physicians:

\section{Extract 9. A nurse describes collaboration with physicians.}

Nurse: It's important that physicians also listen to nurses during ward rounds because nurses are able to inform them about changes in patients' health. 
Based on both the interviews and observations, some physicians did pursue interactional equality and interprofessionalism during ward rounds. However, as mentioned, the quality and quantity of interaction seemed to vary significantly depending on the person and the medical speciality. Extract 10 from the field notes illustrates a situation of interaction between a nurse and physicians:

\section{Extract 10. A nurse and a group of physicians during a ward round}

A group of physicians and a nurse enter the corridor and the nurse starts asking a question regarding a psychiatrist's consultation. The question is evidently interrupted by a discussion between the physicians. The question is heard, however, as physician 1 answers it and gives contact information, physician 2 dictates notes and physician 1 continues to inform the nurse about the patient's subsequent treatment.

Based on the interviews, a shared expectation among the professionals was a smooth exchange of information, fluent collaboration and flexibility. However, again, flexibility and fluent collaboration were understood and realised differently: to physicians, fluent collaboration principally meant obtaining medical information efficiently, whereas to nurses it meant understanding and reconciling overlaps in schedules and appreciating nurses' professional opinion.

The views of the different professionals relating to the challenges and developmental needs of ward rounds also diverged. From the viewpoint of nurses and secretaries, the challenges of ward rounds were largely attributable to schedules and interaction, whereas from the viewpoint of physicians, the challenges related mainly to medicine and data systems. Based on the observations, all the professionals valued swiftness, which was also mentioned as an essential feature of ward rounds in the interviews. Physicians, in particular, are expected to work swiftly. As a whole, based on both the observations and the interviews, synchronising collaboration (different views and work practices) seemed to emerge as a central issue.

\section{Discussion}

Overall, the ward rounds on the EIW were quite physician- and medicine-centred (see e.g. Extract 7), which is congruent with the findings of previous research (e.g. Manias and Street, 2001; Reeves et al., 2009; Nugus et al., 2010). This is partly explained by the fact that the aims of emergency care in general are a fast initial diagnosis, commencement of treatment and quick transfers to appropriate continuing treatment units. Further, the differing aims set for ward rounds naturally reflected the distinct, statutory tasks of the different professional groups. The professionals in this study basically agreed on the main contents of ward rounds (exchange of information and advancement of treatment processes) and on the need for flexibility and fluent collaboration, but views on the challenges of ward rounds and expectations of other professional groups partly diverged. 
Further, despite the basic consensus among the professionals on the main contents of ward rounds, the concept of collaboration was understood differently, especially between physicians and nurses (see e.g. Extracts 7-10), which was also noted by Krogstad and colleagues (2004). Boundaries between medicine and nursing - and the need to further break these boundaries down - were also remarked by Hoskins (2012). To physicians in this study, fluent collaboration mostly denoted obtaining medical information efficiently, which conflicted with nurses' idea of fluent collaboration (interactionally equal collaboration where both medical and non-medical information is exchanged). Nurses did not always actively participate in the conversation between physicians during ward rounds, but the key issue was indeed the possibility of being present and being informed (e.g. having physicians phone nurses as a ward round progressed). Sometimes nurses did want to actively participate in the care planning but were disregarded by physicians (see Extract 7), also noticed by Bradfield (2010). The role of secretaries in the ward rounds seemed rather small: they only participated in the ward rounds of one medical speciality and their main task was to phone and inform nurses on the progress of a ward round. Taking into account the hectic pace of emergency work, one may question (and so did the secretaries themselves) the necessity of the secretaries' presence during ward rounds (see Extracts 5 and 6). More important for them was that physicians dictate their notes from ward rounds swiftly and deliver them to the secretaries immediately (see Extract 6).

For the most part, the ward rounds on the EIW were not interprofessional; the quality and quantity of collaboration depended largely on which physician(s) were participating in the ward rounds. The differences found in this study in the perceptions and actions of professionals regarding the implementation of ward rounds might arise from different understandings of work processes, the distinct statutory responsibilities and the cultures and separate natures of the different professional groups in terms of e.g. education and management - as Wilbur (2014) points out, education and health care systems tend to operate profession-specifically, yet paradoxically professionals are expected to work as a wellfunctioning team when treating patients. However, statutory responsibilities are identical for all members of a given professional group, due to which individual differences, particularly in circumstances lacking mutually composed, consistent guidelines, could account for the variability in the quality and quantity of collaboration found in this study.

Bearing in mind the operational aim of the EIW and emergency care processes in general, it is highly important that patients can be quickly transferred to long-stay wards or discharged. An essential part of implementing this aim is ward rounds, which are complicated by the diverging professional views and expectations, variable work practices and interactional inequality that emerged in this study. Also, ward rounds - a traditional work practice and structure of the hospital context - are challenging when implemented in a fluctuating emergency-natured environment, as opposed to a more traditional hospital ward with perhaps less need for haste and fewer pressures to transfer patients forward. Interprofessional collaboration cannot always be developed by merely adding it to historical structures and work practices; sometimes the structures themselves first need broader modifications to enable the development of interprofessional collaboration. Further, despite the development 
of data systems, face-to-face interaction during ward rounds remains indispensable to ensure patient safety, and for this reason, interaction should be built to be satisfying to all those involved.

This case study contributed to filling research gaps concerning both context-specific studies of collaboration, in this case emergency care, and ward rounds. The findings, although not generalisible due to the small size of the data, can also be utilised in studying and developing emergency-care contexts. Based on our data and suggestions by staff members, we suggest the following developmental ideas for ward rounds: the starting times of ward rounds could be staggered so the nursing staff would be able to participate better, and ward rounds could always proceed in the same order and start from the same room. These changes in particular would clarify the implementation and help in the synchronisation of work tasks, which was an issue, especially for nurses and secretaries.

Owing to the time pressures and the nature of work on the EIW, the demand for swiftness falls particularly upon physicians, but also upon nurses and secretaries. A swift work pace presupposes fluent collaboration and good coordination of tasks: to support this, we suggest that well-functioning collaborative practices be mutually documented. Naturally, people more willingly commit to something they have had a say in. The mutual documentation of collaborative work practices might also clarify the structure of ward rounds, which, in turn, may make it easier for staff to focus on building mutual interaction between staff and patients (see e.g. Weber et al., 2007; O’Leary et al., 2010). Hopefully, this would also diminish some of the negative effects of individual differences on ward round work practices.

Diverging views on interprofessionalism (Copnell et al., 2004; Krogstad et al., 2004) and interactional inequality between professional groups (Bradfield, 2010), both of which also emerged in this study, complicate the organising of genuinely interprofessional rounds because a shared understanding of aims and work practices is pivotal in interprofessionalism (D’Amour et al., 2005; San Martín-Rodriguez et al., 2005). Interprofessionalism is not necessarily ideal if the prevailing (multi-professional) practices of collaboration function well (Collin et al., 2012; 2015). However, in this study, nurses and secretaries in particular expressed their dissatisfaction with many of the current ward round work practices. Interaction might, indeed, often appear unproblematic to the party dominating it (Krogstad et al., 2004), in this case, to physicians.

Interprofessionalism entails the profound exchange and appreciation of different professional opinions, which in turn facilitates the building of a shared view of work processes. A better understanding of each other's work and viewpoints enhances comprehensive and efficient patient care and helps build an open work climate and mutual trust (see San MartínRodriguez et al., 2005; Baker et al., 2006). Therefore, occasions (separate from the hectic everyday work) for getting acquainted with one's colleagues would be beneficial (Collin et al., 2015).

The credibility of the study 
In studies informed by ethnography, central to credibility is how well the viewpoint of participants has been captured in relation to the studied phenomenon (e.g. Hammersley and Atkinson, 2007). In this study, the object of investigation and comparison were the views and actions of members from three different professional groups - physicians, nurses and secretaries - regarding ward rounds. The number of interviewees was rather small, and there were only two interviews with physicians in the data. However, differences in views and actions between the different professionals did clearly emerge in the findings. Indeed, the decisive factor regarding validity in qualitative research is not sample size but the information richness of the selected cases and the observational and analytical capabilities of the researcher (Patton, 2002). We feel that the data were information-rich and, in addition, similar findings were already repeated (Patton, 2002), so we argue that the sample selection is justified. Further, we acknowledge that a fully ethnographic methodology would have required a longer period of observations and more professionals being observed. Credibility was, however, enhanced by three types of triangulation: data, investigator and methodological (Patton, 2002). Observations helped in trying to understand the viewpoint of the interviewees, and the interviews in turn clarified the observations. The discussion between the researchers and the EIW staff in their development session proved an important complement to the interviews and verified the observations and interpretations made from them by the researchers.

\section{Suggestions for further research}

We suggest that more context-specific studies on interprofessional collaboration be conducted in order to build a firm foundation for a general model of shared expertise and organisational development. One interesting aspect in terms of collaboration would also be to study different medical specialities to make different kinds of work practices visible and enhance possibilities to learn from one another. Further, the concept of work process knowledge (Boreham, 2004) - the idea of all employees understanding the whole of the work in a similar way (Boreham, 2004; Pullon, 2008) - has been seen as an interesting angle in attempting to understand collaborative work processes. Professionals' different perceptions on collaboration might, indeed, also proceed from different understandings of work processes. We argue that this concept is also worth exploring in future research.

\section{Acknowledgements}

This work has been supported by the Finnish Work Environment Fund (project number 109295). 


\section{References}

Baker, D.P., Day, R. and Salas, E. (2006), “Teamwork as an essential component of highreliability organizations”, Health Services Research, Vol. 41 No. 4 Part II, pp. 15771598.

Boreham, N. (2004), "Orienting the work-cased curriculum towards work process knowledge: a rationale and a German case study”, Studies in Continuing Education, Vol. 26 No. 2, pp. 209-227.

Bradfield, O.M. (2010), “Ward rounds: the next focus for quality improvement?”, Australian Health Review, Vol. 34 No. 2, pp. 193-196.

Braun, V. and Clarke, V. (2006), "Using thematic analysis in psychology”, Qualitative Research in Psychology, Vol. 3 No. 2, pp. 77-101.

Brewer, J. (2000), Ethnography, Open University Press, Buckingham.

Collin, K., Paloniemi, S. and Herranen, S. (2015), ”INPROF - Promoting teamwork processes and interprofessional collaboration in emergency work (2010-2012)”, Studies in Continuing Education Special Issue. DOI: 10.1080/0158037X.2014.997199

Collin, K., Valleala, U.M., Herranen, S. and Paloniemi, S. (2012), “Ways of interprofessional collaboration and learning in emergency work”, Studies in Continuing Education, Vol. 34 No. 3, pp. 281-300.

Copnell, B., Johnston, L., Harrison, D., Wilson, A., Robson, A., Mulcahy, C., Ramudu, L., McDonnell, G. and Best, C. (2004), “Physicians' and nurses' perceptions of interdisciplinary collaboration in the NICU, and the impact of a neonatal nurse practitioner model of practice”, Journal of Clinical Nursing, Vol. 13 No. 1, pp. 105113.

Creamer, G.L., Dahl, A., Perumal, A., Tan, G. and Koea, J.B. (2010), “Anatomy of the ward round: the time spent in different activities”, ANZ Journal of Surgery, Vol. 80 No. 12, pp. 930-932.

D’Amour, D., Ferrada-Videla, M., San Martín-Rodriguez, L. and Beaulieu, M-D. (2005), "The conceptual basis for interprofessional collaboration: core concepts and theoretical frameworks”, Journal of Interprofessional Care, Vol. 19 No. 2, pp. 116-131.

Fiddler, M., Borglin, G., Galloway, A., Jackson, C., McGowan, L. and Lovell, K. (2010), “Once-a-week psychiatric ward round or daily inpatient team meeting? A multidisciplinary mental health team's experience of new ways of working”, International Journal of Mental Health Nursing, Vol. 19 No. 2, pp. 119-127. 
Gordon, T., Hynninen, P., Lahelma, E., Metso, T., Palmu, T. and Tolonen, T. (2006), “Collective ethnography, joint experiences and individual pathways”, Nordisk Pedagogik [Journal of Nordic Educational Research], Vol. 26 No. 1, pp. 3-15.

Hammersley, M. and Atkinson, P. (2007), Ethnography. Principles in practice, Taylor \& Francis, London.

Hoskins, R. (2012), “Interprofessional working or role substitution? A discussion of the emerging roles in emergency care”, Journal of Advanced Nursing, Vol. 68 No. 6, pp. 1894-1903.

Housley, W. (2003), Interaction in multidisciplinary teams, Ashgate, Aldershot.

Krogstad, U., Hofoss, D. and Hjortdahl, P. (2004), ”Physician and nurse perception of interprofessional co-operation in hospitals”, International Journal for Quality Health Care, Vol. 16 No. 6, pp. 491-497.

Lemieux-Charles, L. and McGuire, W. (2006), "What do we know about health care team effectiveness: a review of literature”, Medical Care Research and Review, Vol. 63 No. 3, pp. 1-38.

MacNaughton, K., Chreim, S. and Bourgeault, I.L. (2013), "Role construction and boundaries in interprofessional primary health care teams: a qualitative study”, BMC Health Services Research, Vol. 13, pp. 486-498.

Manias, E. and Street, S. (2001), “Nurse-physician interactions during critical care ward rounds”, Journal of Clinical Nursing, Vol. 10 No. 4, pp. 442-450.

McCallin, A. (2001), "Interdisciplinary practice - a matter of teamwork: an integrated literature review”, Journal of Clinical Nursing, Vol. 10 No. 4, pp. 419-428.

Millward, L.J. and Jeffries, N. (2001), “The team survey: a tool for health care team development”, Journal of Advanced Nursing, Vol. 35 No. 2, pp. 276-287.

Nembhard, I.M. and Edmondson, A.C. (2006), "Making it safe: the effects of leader inclusiveness and professional status on psychological safety and improvement efforts in health care teams”, Journal of Organizational Behavior, Vol. 27 No. 7, pp. 941-966.

Nugus, P., Greenfield, D., Travaglia, J., Westbrook, J. and Braithwaite, J. (2010), “How and where clinicians exercise power: interprofessional relations in health care”, Social Science \& Medicine, Vol. 71 No. 5, pp. 898-909.

O’Leary, K.J., Wayne, D.B., Haviley, C., Slade, M.E., Lee, J. and Williams, M.V. (2010), "Improving teamwork: impact of structured interdisciplinary rounds on a hospitalist unit”, Journal of General Internal Medicine, Vol. 25 No. 8, pp. 826-832. 
Patton, M. Q. (2002), Qualitative research \& evaluation methods, $3^{\text {rd }}$ ed., Thousand Oaks, Sage.

Pisano, G.P., Bohmer, R.M.J. and Edmondson, A.C. (2001), “Organizational differences in rates of learning: evidence from the adoption of minimally invasive cardiac surgery”, Management Science, Vol. 47 No. 6, pp. 752-768.

Pullon, S. (2008), “Competence, respect and trust: key features of successful interprofessional nurse-physician relationships”, Journal of Interprofessional Care, Vol. 22, No. 2, pp. 133-147.

Ramanujam, R. and Rousseau, D.M. (2006), “The challenges are organizational, not just clinical”, Journal of Organizational Behavior, Vol. 27 No. 7, pp. 811-827.

Reeves, S., Rice, K., Conn, L.G., Miller, K-L., Kenaszchuk, C. and Zwarenstein, M. (2009), "Interprofessional interaction, negotiation and non-negotiation on general internal medicine wards”, Journal of Interprofessional Care, Vol. 23 No. 6, pp. 633-645.

San Martín-Rodriguez, L., Beaulieu, M.D., D’Amour, D. and Ferrada-Videla, M. (2005), "The determinants of successful collaboration: a review of theoretical and empirical studies”, Journal of Interprofessional Care, Vol. 19 No. 2, pp. 132-147.

Sigaud, L. (2008), “A collective ethnographer: fieldwork experience in the Brazilian Northeast”, Social Science Information, Vol. 47 No. 1, pp. 71-97.

Walton, J.M. and Steinert, Y. (2010), “Patterns of interaction during rounds: implications for work-based learning”, Medical Education, Vol. 44 No. 6, pp. 550-558.

Weber, H., Stöckli, M., Nübling, M. and Langewitz, W.A. (2007), ”Communication during ward rounds in Internal Medicine. An analysis of patient-nurse-physician interactions using RIAS”, Patient Education and Counseling, Vol. 67 No. 3, pp. 343-348.

Wilbur, L. (2014), "Interprofessional education and collaboration: A call to action for emergency medicine”, Academic Emergency Medicine, Vol. 21 No. 7, pp. 833-834.

Woods, P., Boyle, M., Jeffrey, B. and Troman, G. (2000), “A research team in ethnography”, International Journal of Qualitative Studies in Education, Vol. 13 No. 1, pp. 85-98. 